\title{
Risk of dementia after anaesthesia and surgery
}

Pin-Liang Chen, ${ }^{*}$ Chih-Wen Yang, * Yi-Kuan Tseng, Wei-Zen Sun, Jane-Ling Wang, Shuu-Jiun Wang, Yen-Jen Oyang and Jong-Ling Fuh

\section{Background}

The potential relationship between anaesthesia, surgery and onset of dementia remains elusive.
Aims
To determine whether the risk of dementia increases after surgery with anaesthesia, and to evaluate possible associations among age, mode of anaesthesia, type of surgery and risk of dementia.

\section{Method}
The study cohort comprised patients aged 50 years and older who were anaesthetised for the first time since 1995 between 1 January 2004 and 31 December 2007, and a control group of randomly selected patients matched for age and gender. Patients were followed until 31 December 2010 to identify the emergence of dementia.

\section{Results}

Relative to the control group, patients who underwent anaesthesia and surgery exhibited an increased risk of dementia (hazard ratio $=1.99$ ) and a reduced mean interval to dementia diagnosis. The risk of dementia increased in patients who received intravenous or intramuscular anaesthesia, regional anaesthesia and general anaesthesia.

\section{Conclusions}

The results of our nationwide, population-based study suggest that patients who undergo anaesthesia and surgery may be at increased risk of dementia.

\section{Declaration of interest}

None.
Generally considered safe and effective, anaesthetic drugs have bestowed enormous clinical benefits. However, there is growing concern that they may have neurodegenerative complications. In vitro and multidimensional nuclear magnetic resonance studies have shown that inhaled anaesthetic agents can promote amyloid $\beta$ peptide $(A \beta)$ oligomerisation and enhance $A \beta$-induced neurotoxicity. ${ }^{1,2}$ Animal studies also provide evidence that exposure to inhaled anaesthetic drugs can impair memory, ${ }^{3}$ and induce caspase-3 activation and increased levels of $\mathrm{A} \beta .^{4,5}$ Others have identified calcium dysregulation as a possible mechanism of anaesthetic-induced neurotoxicity. ${ }^{6}$ Post-operative cognitive decline in humans is generally considered to be a short-lived condition with normal function returning within days, but cognitive changes may persist for weeks or more. ${ }^{7}$ It is not yet clear whether this decline represents an unmasking of early dementia or a predictor of later dementia. Regardless, with clinical features similar to those observed in people with dementia, postoperative long-term cognitive impairment raises concerns that anaesthesia and surgery may accelerate the onset and progression of neurodegenerative dementia. ${ }^{8}$ Whether anaesthesia and surgery contribute to the development of long-term cognitive decline, however, remains controversial. One study showed a decline in cognitive function 5 years after coronary arterial bypass graft (CABG) operations, ${ }^{9}$ and a retrospective study showed that patients who underwent CABG had a higher incidence of Alzheimer's disease in the following $5-6$ years. ${ }^{8}$ However, another study found that similar proportions of Alzheimer's disease and control group patients had been subjected to CABG operations. ${ }^{10}$ Moreover, others have reported no link between anaesthesia, surgery and long-term cognitive decline. ${ }^{10,11}$ It is possible that small cohorts, biased study populations and confounding coincident illnesses may have contributed to the contradictory results. ${ }^{10}$ Thus, the consensus statement from the First International Workshop on Anesthetics and Alzheimer's Disease in 2009 suggested that statistically sound prospective and

*These authors contributed equally to this work. retrospective human studies of the risk of Alzheimer's disease after anaesthesia are needed. ${ }^{12}$

The Taiwan National Health Insurance Research Database (NHIRD) provides data from a large and representative Taiwanese population that has been followed for over a decade. In this study we analysed the NHIRD retrospectively to determine whether the risk of neurodegenerative dementia increases after anaesthesia and surgery, and to evaluate possible associations between the risk of dementia and patient age, mode of anaesthesia and type of surgery.

\section{Method}

At the time of our analysis the NHIRD contained records for approximately 23 million enrolled persons dating back to March 1995, representing almost $99 \%$ of the total population of Taiwan (http://nhird.nhri.org.tw/en). We reviewed records from the Longitudinal Health Insurance Database (LHID), which includes records derived from the NHIRD by systematic random sampling. The LHID includes claims data for 1 million patients. We observed no significant difference in gender, age or average income distributions between the LHID and the NHIRD.

\section{Study sample}

We extracted the records of patients aged 50 years or over who underwent anaesthesia for the first time since 1995 between 1 January 2004 and 31 December 2007, based on the ICD-9 Clinical Modification (ICD-9-CM) codes. ${ }^{13}$ To avoid the influence of chemotherapy and brain metastasis, we excluded patients who had any diagnosis of cancer (ICD-9-CM 140-208). Similarly, we excluded patients with a history of dementia, Parkinsonism (ICD-9-CM 332), stroke (ICD-9-CM 430-434) or brain operations (ICD-9-CM operative codes 01-04). We tracked each individual in the study cohort from the date of anaesthesia until 31 December 2010. The control cohort was selected from the remaining patients who had not received anaesthesia, excluding 
those with a history of cancer, dementia, Parkinsonism or stroke. For each individual in the anaesthesia group we selected 4 or 5 control patients randomly, but matched for exact age and gender.

\section{Events}

The first occurrence of dementia diagnosis was identified. We accepted the diagnoses of presenile dementia, senile dementia (ICD-9-CM 290.0-290.3) and Alzheimer's dementia (ICD-9-CM 331.0 ) as forms of neurodegenerative dementia. Such diagnoses were established mostly by board-certified neurologists or psychiatrists after routine blood count, blood chemistry, thyroid hormone, folate, vitamin $\mathrm{B}_{12}$, syphilis and neuropsychological tests and brain imaging (computed tomography or magnetic resonance imaging) to exclude dementia due to other causes and accepted in light of applications for reimbursement for dementia prescription medication. Because perioperative stroke is a surgical complication that could potentially contribute to the development of vascular dementia, we excluded patients with a diagnosis of atherosclerotic dementia (ICD-9-CM 290.4). In contrast to post-operative cognitive decline, a transient and reversible condition, dementia is a slowly developing and progressive neurodegenerative process. Therefore, patients were considered eligible for the study cohort if a diagnosis of dementia had been recorded at least twice, with the first diagnosis occurring at least 3 months after the first anaesthesia experience.

\section{Covariates}

We extracted demographic information, including age, gender and the following potentially confounding factors: hypertension (ICD-9-CM 401), hyperlipidaemia (ICD-9-CM 272) and depression (ICD-9-CM 296.2-296.3, 300.4, 311). Among patients with depression, those treated by electroconvulsive therapy (ECT; ICD-9-CM procedure codes 9426, 9427) were identified. We calculated the Charlson index to account for 22 comorbidities (e.g. myocardial infarction and diabetes mellitus) using a total score that was weighted according to the presence of the various conditions. ${ }^{14}$ To assess the differences in the risks of dementia based on covariates, we divided the sample into two age categories: middle-aged (50-65 years) and senior ( $>65$ years). The modes of anaesthesia received were classified into three groups: intravenous or intramuscular (IV/IM) anaesthesia, regional anaesthesia and general anaesthesia. Patients in the IV/ IM group typically received combined regimens that included propofol, midazolam, thiopental or ketamine as sedatives for operations that were relatively short in duration. The regional anaesthesia group included patients who had been given epidural, spinal or local anaesthesia. The general anaesthesia group included patients who were given hypnotics such as propofol, barbiturates or etomidate as sedatives prior to administration of inhaled or IV anaesthetic drugs for maintenance. The number of separate anaesthesia treatments that participants received within a year was used as a covariate, with one level of the covariate indicating a single exposure to anaesthesia and a second level indicating two or more exposures. We also grouped patients according to the type of surgery they received (ICD-9-CM operative codes in parentheses): eye surgery (08-16), ear, nose and throat (ENT) surgery (18-29), respiratory surgery (30-34), cardiovascular surgery (35-39), digestive surgery (42-54), genitourinary surgery (55-71), musculoskeletal surgery (76-84) or dermatological surgery (86). All surgical groups were mutually exclusive.

\section{Statistical analysis}

All statistical analyses were performed with the SAS statistical software package for Windows version 9.2. Clinical variables were compared between study and control groups using the chi-squared test. Cox regression was used to estimate hazard ratios (HRs) and 95\% confidence intervals. We adjusted the models for possible confounding factors, including hypertension, hyperlipidaemia, depression and the Charlson index; the values of the confounding factors were at baseline. All tests were two-tailed, and a $P$-value of less than 0.05 was considered statistically significant.

\section{Results}

A total of 24901 patients were included in the anaesthesia group and 110972 in the control group. Relative to the control group, those in the anaesthesia group were slightly older (63 years $v .61$ years, $P<0.001)$ and more frequently men $(49.5 \%$ v. $48.2 \%$, $P<0.001$ ) (Table 1). The prevalence of comorbidity, including hypertension, hyperlipidaemia and depression, was higher in the anaesthesia group $(P<0.001)$. The Charlson index was also higher in the anaesthesia group (mean 2.15 v. 1.49, $P<0.001$ ).

General anaesthesia was the most commonly used mode of anaesthesia $(55.1 \%)$, followed by regional anaesthesia $(35.2 \%)$ and IV/IM anaesthesia (6.8\%) (Table 2); $42.9 \%$ of the anaesthesia group received anaesthesia at least twice within a single year. The most frequent surgery types were musculoskeletal (39.5\%), digestive $(20.5 \%)$ and genitourinary (16.3\%). A summary of the

\begin{tabular}{|c|c|c|c|}
\hline & $\begin{array}{l}\text { Anaesthesia group } \\
\qquad(n=24901)\end{array}$ & $\begin{array}{l}\text { Control group } \\
(n=110972)\end{array}$ & $P^{\mathrm{a}}$ \\
\hline Age, years: median (IQR) & $63(55-71)$ & $61(54-69)$ & $<0.001$ \\
\hline \multicolumn{4}{|l|}{ Age group, $n(\%)$} \\
\hline 50-65 years & $14248(57.2)$ & 70957 (63.9) & \\
\hline$>65$ years & $10653(42.8)$ & $40015(36.1)$ & \\
\hline Male, $n(\%)$ & $12332(49.5)$ & $53528(48.2)$ & $<0.001$ \\
\hline \multicolumn{4}{|l|}{ Comorbidities, $n$ (\%) } \\
\hline Hypertension & $10501(42.2)$ & 37955 (34.2) & $<0.001$ \\
\hline Hyperlipidaemia & 7084 (28.4) & $26289(23.7)$ & $<0.001$ \\
\hline Depression & $1046(4.2)$ & $3044(2.7)$ & $<0.001$ \\
\hline \multicolumn{4}{|l|}{ Charlson index } \\
\hline Mean score & 2.15 & 1.49 & $<0.001$ \\
\hline$<3, n(\%)$ & $16080(64.6)$ & $86892(78.3)$ & \\
\hline$\geqslant 3, n(\%)$ & $8821(35.4)$ & $24080(21.7)$ & \\
\hline
\end{tabular}




\begin{tabular}{|lc|}
$\begin{array}{l}\text { Table } 2 \text { Anaesthesia group: anaesthesia and type of surgery } \\
(n=24901)\end{array}$ & $n(\%)$ \\
\hline & \\
\hline & \\
Mode of anaesthesia ${ }^{a}$ & $1686(6.8)$ \\
IV or IM & $8777(35.2)$ \\
Regional & $13715(55.1)$ \\
\hline General & \\
\hline Number of times anaesthesia given within 1 year & $14212(57.1)$ \\
Once & $10689(42.9)$ \\
\hline Twice or more & $1206(4.8)$ \\
\hline Type of surgery & $1182(4.7)$ \\
Eye & $425(1.7)$ \\
ENT & $950(3.8)$ \\
Respiratory & $5108(20.5)$ \\
Cardiovascular & $4062(16.3)$ \\
Digestive & $9825(39.5)$ \\
Genitourinary & $762(3.1)$ \\
Musculoskeletal & \\
Dermatological & \\
ENT, ear, nose and throat; IM, intramuscular; IV, intravenous. \\
a. Some modes of anaesthesia (e.g. nitrous oxide-oxygen sedation, hypothermia \\
anaesthesia) are not shown owing to small numbers. \\
b. Some types of surgery (e.g. endocrine) are not shown owing to small numbers. \\
\hline
\end{tabular}

distribution of anaesthesia type in relation to surgery type is provided in Table 3.

During 3-7 years of follow-up, 661 of the 24901 (2.65\%) anaesthesia group patients and 1539 of the $110972(1.39 \%)$ control group patients were diagnosed with dementia. The mean duration of dementia diagnosis from the date of the first anaesthesia exposure was shorter in the anaesthesia group than in the control group (906.7 days, s.d. $=623.5$, v. 1104.3 days, s.d. $=$ 609.8, $P<0.0001$ ). Adjusting for hypertension, hyperlipidaemia, depression and Charlson index yielded an estimated 1.99-fold increased risk for developing dementia in the anaesthesia group (95\% CI 1.81-2.17, $P<0.001)$. The risk of dementia after anaesthesia was increased similarly in men $(\mathrm{HR}=2.30,95 \% \mathrm{CI} 2.00-2.65)$ and women $(\mathrm{HR}=1.80,95 \%$ CI 1.60-2.03). Significant associations between the emergence of dementia and exposure to anaesthesia were observed in both middle-aged $(\mathrm{HR}=1.90$, 95\% CI 1.49 2.42) and senior patients ( $\mathrm{HR}=1.79,95 \%$ CI 1.62-1.97). The increased risk of dementia was observed for patients with a Charlson index less than 3 ( $\mathrm{HR}=1.90,95 \% \mathrm{CI} 1.68-2.16)$, as well as for those with an index greater than $3(\mathrm{HR}=1.67,95 \%$ CI 1.45 1.91) (Table 4). Among the patients with depression, the hazard ratio of dementia after anaesthesia and surgery was 3.59 (95\% CI 2.58-5.00); those with depression diagnosed after anaesthesia
Table 3 Distribution of the three types of anaesthesia among different types of surgical procedures

\begin{tabular}{|lccc|} 
& \multicolumn{3}{c}{ Distribution, \% } \\
\cline { 2 - 4 } Surgery & IV or IM & Regional & General \\
\hline Eye & 2.0 & 73.5 & 24.5 \\
\hline ENT & 0.9 & 0.0 & 99.1 \\
\hline Respiratory & 5.4 & 0.2 & 94.4 \\
\hline Cardiovascular & 8.7 & 9.7 & 81.6 \\
\hline Digestive & 7.7 & 37.0 & 55.3 \\
\hline Genitourinary & 6.9 & 51.5 & 41.6 \\
\hline Musculoskeletal & 5.3 & 36.8 & 57.9 \\
\hline Dermatological & 19.7 & 33.5 & 46.8 \\
\hline ENT, ear, nose and throat; IM, intramuscular; IV, intravenous. & \\
\hline
\end{tabular}

and surgery had a higher hazard ratio than those with depression before the procedure ( $\mathrm{HR}=4.79,95 \%$ CI 3.09-7.43, $v . \mathrm{HR}=2.51$, 95\% CI 1.50-4.18). However, of the $18(1.7 \%)$ individuals in the anaesthesia group with a diagnosis of depression $(n=1046)$ who received ECT, none developed dementia.

Table 5 shows the associations of dementia with mode of anaesthesia and number of anaesthesia treatments within a single year. The adjusted hazard ratio of incident dementia was greatest in patients who received regional anaesthesia $(\mathrm{HR}=1.80,95 \% \mathrm{CI}$ $1.57-2.07)$, followed by IV/IM anaesthesia $(\mathrm{HR}=1.60,95 \% \mathrm{CI}$ $1.11-2.30)$ and general anaesthesia $(\mathrm{HR}=1.46,95 \%$ CI $1.28-$ 1.68). Compared with the general anaesthesia group, the regional anaesthesia group - but not the IV/IM anaesthesia group - had a higher risk of dementia ( $\mathrm{HR}=1.42,95 \%$ CI 1.20-1.66). Exposure to anaesthesia at least twice within a year yielded a 1.75 -fold increased risk of dementia (95\% CI 1.53-2.01), whereas exposure to anaesthesia only once within a year yielded a 1.73 -fold increased risk of dementia (95\% CI 1.53-1.96), with no significant difference in risk between these two groups.

Five of the eight types of surgery were associated with an increased risk of dementia: these were dermatological, musculoskeletal, genitourinary, digestive and eye surgery (Table 6). In contrast, the incidences of dementia after ENT, respiratory and cardiovascular surgery did not differ significantly from the incidence observed for the control group.

\section{Discussion}

We found an almost doubled risk of development of dementia within 3-7 years of anaesthesia and surgery. The mean duration

\begin{tabular}{|c|c|c|c|c|c|}
\hline Group & $\begin{array}{c}\text { Patients with dementia diagnosis } \\
\text { in the anaesthesia group } \\
n(\%)\end{array}$ & $\begin{array}{c}\text { Patients with dementia } \\
\text { diagnosis in the control group } \\
n(\%)\end{array}$ & $\operatorname{HR}(95 \% \mathrm{Cl})$ & Adjusted HR $(95 \% \mathrm{Cl})^{\mathrm{a}}$ & $P$ \\
\hline All & $661(2.65)$ & 1539 (1.39) & $1.99(1.81-2.17)$ & $1.75(1.59-1.92)$ & $<0.001$ \\
\hline \multicolumn{6}{|l|}{ Gender } \\
\hline Male & $296(2.40)$ & 583 (1.09) & $2.30(2.00-2.65)$ & $2.01(1.78-2.37)$ & $<0.001$ \\
\hline Female & $365(2.90)$ & $956(1.66)$ & $1.80(1.60-2.03)$ & $1.58(1.40-1.78)$ & $<0.001$ \\
\hline \multicolumn{6}{|l|}{ Age, years } \\
\hline $50-65$ & $91(0.64)$ & $239(0.34)$ & $1.90(1.49-2.42)$ & $1.65(1.30-2.11)$ & $<0.001$ \\
\hline$>65$ & $570(5.35)$ & $1300(3.25)$ & $1.79(1.62-1.97)$ & $1.70(1.53-1.87)$ & $<0.001$ \\
\hline \multicolumn{6}{|c|}{ Charlson index } \\
\hline$<3$ & $91(0.57)$ & $293(0.34)$ & $1.90(1.68-2.16)$ & $1.85(1.63-2.09)$ & $<0.001$ \\
\hline$\geqslant 3$ & $570(6.46)$ & $1246(5.17)$ & $1.67(1.45-1.91)$ & $1.63(1.43-1.87)$ & $<0.001$ \\
\hline
\end{tabular}


Table 5 Hazard ratios of dementia in the anaesthesia group in terms of mode of anaesthesia and number of times anaesthesia administered within 1 year ${ }^{a}$

\begin{tabular}{|c|c|c|c|c|c|c|}
\hline & $\begin{array}{c}\text { Patients with dementia diagnosis } \\
\text { in the anaesthesia group } \\
n(\%)\end{array}$ & $\begin{array}{c}\text { Patients with dementia } \\
\text { diagnosis in the control group } \\
n(\%)\end{array}$ & $\begin{array}{l}\text { Adjusted HR } \\
\quad(95 \% \mathrm{Cl})\end{array}$ & $P$ & $\begin{array}{l}\text { Adjusted HR } \\
(95 \% \mathrm{Cl})\end{array}$ & $P$ \\
\hline \multicolumn{7}{|c|}{ Mode of anaesthesia ${ }^{b}$} \\
\hline IV or IM & $45(2.67)$ & $97(1.27)$ & $1.60(1.11-2.30)$ & 0.011 & $1.07(0.78-1.47)^{\mathrm{C}}$ & 0.664 \\
\hline Regional & $299(3.41)$ & 643 (1.69) & $1.80(1.57-2.07)$ & $<0.001$ & $1.42(1.20-1.66)^{C}$ & $<0.001$ \\
\hline General & 301 (2.19) & $778(1.26)$ & $1.46(1.28-1.68)$ & $<0.001$ & 1.00 & \\
\hline \multicolumn{7}{|l|}{ Frequency $^{d}$} \\
\hline Once & $357(2.51)$ & $863(1.36)$ & $1.73(1.53-1.96)$ & $<0.001$ & 1.00 & \\
\hline Twice or more & $304(2.84)$ & $676(1.43)$ & $1.75(1.53-2.01)$ & $<0.001$ & $1.09(0.93-1.27)^{\mathrm{e}}$ & 0.290 \\
\hline \multicolumn{7}{|c|}{$\begin{array}{l}\text { IM, intramuscular; IV, intravenous; HR, hazard ratio. } \\
\text { a. All models are analysed by Cox regressions adjusted for hypertension, hyperlipidaemia, depression and Charlson index. } \\
\text { b. Some modes of anaesthesia (e.g. nitrous oxide-oxygen sedation, hypothermia anaesthesia) are not shown owing to small numbers. } \\
\text { c. Group comparison with general anaesthesia as baseline. } \\
\text { d. Number of times anaesthesia administered within } 1 \text { year. } \\
\text { e. Group comparison with anaesthesia exposure once within } 1 \text { year as baseline. }\end{array}$} \\
\hline
\end{tabular}

\begin{tabular}{|c|c|c|c|c|}
\hline Type of surgery ${ }^{a}$ & $\begin{array}{c}\text { Patients with dementia diagnosis } \\
\text { in the anaesthesia group } \\
n(\%)\end{array}$ & $\begin{array}{c}\text { Patients with dementia } \\
\text { diagnosis in the control group } \\
n(\%)\end{array}$ & $\begin{array}{c}\text { Adjusted HR } \\
(95 \% \mathrm{Cl})^{\mathrm{b}}\end{array}$ & $P$ \\
\hline Eye & $36(2.99)$ & $90(1.70)$ & $1.55(1.05-2.31)$ & 0.029 \\
\hline ENT & $16(1.35)$ & $45(0.80)$ & $1.46(0.82-2.61)$ & 0.198 \\
\hline Respiratory & $5(1.18)$ & $35(2.08)$ & $0.49(0.19-1.27)$ & 0.141 \\
\hline Cardiovascular & $17(1.79)$ & $66(1.62)$ & $0.92(0.53-1.61)$ & 0.771 \\
\hline Digestive & $122(2.39)$ & $284(1.26)$ & $1.75(1.41-2.17)$ & $<0.001$ \\
\hline Genitourinary & 89 (2.19) & 189 (1.03) & $1.93(1.50-2.49)$ & $<0.001$ \\
\hline Musculoskeletal & $330(3.36)$ & 728 (1.68) & $1.88(1.65-2.15)$ & $<0.001$ \\
\hline Dermatological & $27(3.54)$ & $50(1.45)$ & $2.36(1.46-3.80)$ & $<0.001$ \\
\hline
\end{tabular}

of dementia diagnosis was shorter in patients who underwent anaesthesia and surgery than in those in the control group. These findings suggest a significant association between anaesthesia with surgery and subsequent dementia. The increased risk of dementia was independent of age, gender, Charlson index, mode of anaesthesia and number of anaesthesia exposures within a year. In contrast to prior conflicting studies, ${ }^{10}$ in this nationwide, population-based analysis we examined a large number of cases, including four or five age- and gender-matched control individuals for each anaesthesia group patient, and made adjustments for potential confounding factors. Owing to our study design, the present results provide statistically sound evidence for the putative association of dementia with anaesthesia and surgery.

Because the diagnosis of dementia requires the presence of persistent cognitive decline, we used dementia as a marker of clinically significant brain dysfunction to investigate whether anaesthesia would cause long-term detrimental effects on the brain. After excluding vascular dementia and Parkinsonism with dementia, Alzheimer's disease accounted for the majority of dementia cases in our cohort. Therefore, our findings suggest that exposure to anaesthesia with surgery might increase patients' risk of developing Alzheimer's disease. The Taiwanese NHIRD is a nationwide medical claim database, and it is possible that some people with Alzheimer's disease were misdiagnosed. However, it is likely that variability in diagnosis of dementia would be similar in both the anaesthesia and the control groups. Additionally, the long interval between anaesthesia exposure and dementia diagnosis in both our study groups should provide confidence that our results were not confounded by post-operative cognitive decline. To be more certain that cases of post-operative cognitive decline were not included, we considered patients to have developed dementia if their first dementia diagnosis occurred 6 months or 12 months after the anaesthesia. The results still showed that relative to the control group, the anaesthesia group exhibited an increased risk of dementia (data not shown).

\section{Attribution of risk}

Anaesthesia and surgery are inseparable in clinical settings. Thus, it is difficult to establish whether the increased risk of dementia development observed was attributable to anaesthesia per se, the surgical process, or both. It is noteworthy that there is a growing body of laboratory evidence suggesting that anaesthetic agents may interact with Alzheimer's disease neuropathology at multiple levels in the involved pathways. However, human evidence has been lacking. Another plausible mechanism of dementia development after anaesthesia and surgery is the occurrence of perioperative events. To help control for this possibility, we excluded patients with vascular dementia. The association of overt perioperative stroke with dementia was beyond the scope of our study. Nevertheless, microvascular perioperative brain damages, such as white matter lesions and silent small infarctions, might influence the risk of dementia development. Alzheimer's disease has also been linked to hypoxia and hypocapnia events. ${ }^{15}$ Deliberate or unintended hypothermia in the perioperative period might also be a risk factor. ${ }^{12}$ In animal studies, anaesthesia-induced hypothermia has been shown to 
produce tau hyperphosphorylation, a biochemical process that may have a role in Alzheimer's disease pathogenesis. ${ }^{4}$ However, caution must be exercised in asserting causality, because surgery with anaesthesia shares some common features with dementia pathology, such as old age and involvement of inflammatory processes. The other possible explanation for the observed association between surgery under anaesthesia and subsequent dementia is that patients are more susceptible to surgical illness prior to a clinical diagnosis of dementia. Thus, some comorbidities associated with Alzheimer's disease might cause patients to be prone to surgical intervention. ${ }^{16}$ However, it is noteworthy that some illnesses, such as cancer, have been shown to have an inverse association with Alzheimer's disease. ${ }^{17}$ Unfortunately, clinical evidence of susceptibility to surgical illness during the preclinical period of Alzheimer's disease is scant.

The best-identified risk factor for post-operative long-term cognitive decline and Alzheimer's disease is increasing age. ${ }^{18}$ In animal studies, less effect on cognitive function attributable to the anaesthesia was noted in adolescent or middle-aged rodents. ${ }^{19}$ Thus, the older brain may demonstrate enhanced vulnerability to mild insults. As expected, our study showed that the incidence of dementia after anaesthesia with surgery was higher in senior patients than in middle-aged patients. However, the extent of increased risk of dementia was similar between these two groups. One previous study of patients with Alzheimer's disease showed that age at onset was inversely related to cumulative exposure to anaesthesia before the age of 50 years. ${ }^{20}$ These results suggest that anaesthesia and surgery may speed up the process of neurodegeneration, and that this is not specific to older people.

\section{Type of anaesthesia and surgery}

Thus far, most studies whose results support the proposition of anaesthetic-induced neurotoxicity have examined inhaled anaesthetic agents. Limited data are available in relation to the effects of IV anaesthetic drugs, which are generally considered less neurotoxic than inhaled agents. Propofol only enhanced $A \beta$ oligomerisation at high concentrations, ${ }^{1}$ and did not impair memory in rats. ${ }^{21}$ There has been an ongoing debate as to whether regional anaesthesia is superior to general anaesthesia in relation to the incidence of cognitive decline; previous studies have shown inconsistent results. One study demonstrated that $A \beta$ and tau in cerebrospinal fluid changed in a manner consistent with Alzheimer's disease 6 months after CABG, regardless of whether the patient received inhaled or IV anaesthesia. ${ }^{22}$ One study showed that patients exposed to general anaesthesia had a lower risk of dementia 5 years after prostate or hernia surgery than did patients given regional anaesthesia, ${ }^{7}$ but other studies demonstrated no significant difference between these types of anaesthesia. ${ }^{23,24}$ In our study all three modes of anaesthesia were associated with an increased risk of dementia, and the hazard ratio for dementia development was higher in the regional anaesthesia group than in the general anaesthesia group, whereas there was no significant difference between the IV/IM group and the general anaesthesia group. It may be that anaesthetic-induced neurotoxicity does not play a key part in the onset or acceleration of Alzheimer's disease pathogenesis after anaesthesia and surgery. Other perianaesthesia factors, such as duration and depth of anaesthesia, might also modulate Alzheimer's disease pathogenesis. ${ }^{25}$

There is a bidirectional link between depression and dementia. In our study the approximately fourfold increase in dementia risk associated with depression may suggest that depression is a risk factor for development of dementia after anaesthesia and surgery, especially when the diagnosis of depression occurred post-operatively. Nonetheless, this association may also suggest that depression might be a prodrome of dementia. Electroconvulsive therapy is sometimes administered with IV/IM anaesthesia to people with severe depression, and has been reported to cause cognitive decline. ${ }^{26}$ However, none of the 18 patients in our study who had received ECT developed dementia. Therefore, we can conclude that our finding of increased risk of dementia after IV/IM anaesthesia was not biased by inclusion of these patients with depression. On the other hand, given the small number of cases of ECT treatment, we cannot elaborate on the possible association of exposure to IV/IM anaesthesia during ECT with dementia development in patients with depression.

Our data do not support the hypothesis that two or more exposures to anaesthesia within a year increase the risk of Alzheimer's disease development beyond that occurring as a result of a single exposure within a year. Our data are consistent with a prior case-control study showing that neither exposure to six or more episodes of general anaesthesia, nor cumulative exposure to $600 \mathrm{~min}$ or more of general anaesthesia, was associated with an increased risk of Alzheimer's disease. ${ }^{27}$ In contrast, cell culture and animal studies have suggested that inhaled anaesthetic agents induce neuronal apoptosis in a dose- and time-dependent manner. ${ }^{1,6}$ An epidemiological study found that age at onset of Alzheimer's disease was inversely related to cumulative exposure to anaesthesia in earlier life. ${ }^{20}$ The inconsistency between previous studies and our findings may be due to different study designs and different methods of statistical analysis.

With the exception of studies related to cardiac surgery, there is a dearth of research reports providing information about the possible relationship between surgery type and dementia development. ${ }^{8,10}$ In our analysis cardiovascular surgery was not associated with an increased risk of Alzheimer's disease; however, it should be noted that our cohort included only 17 patients who underwent cardiovascular surgery, which limits interpretations of this result. The small number of patients in this subgroup was most probably due to our exclusion of patients with a diagnosis of stroke. A study of non-cardiac surgery reported that the incidence of cognitive dysfunction 1-2 years following major abdominal, thoracic or orthopaedic surgery was similar to that in the control group. ${ }^{28}$ However, the number of participants in the control group might have been inadequate. Another group did not detect long-term cognitive decline attributable to noncardiac surgery, but their analysis did not consider surgery type. ${ }^{29}$ In our study, patients undergoing digestive, genitourinary or musculoskeletal surgery showed an increased risk of dementia. Eye and dermatological surgery were also associated with increased risk of dementia, but the case numbers in these subgroups were small. The effect of the surgery itself on cognitive decline remains unclear. Indeed, in animals, surgery combined with anaesthesia produced greater cognitive dysfunction than anaesthesia alone. ${ }^{30}$ In addition, inflammatory cascades and microglial activation, which are strongly implicated in neurodegeneration, are triggered by surgery. Thus, surgery may initiate a pro-inflammatory event in the brain in both humans and other animals. ${ }^{31,32}$ Moreover, enhanced neuro-inflammation is hypothesised to accelerate Alzheimer's disease neuropathology. ${ }^{31}$

\section{Limitations of the study}

Several caveats merit attention in the interpretation of our results. Because we drew our source data from the NHIRD claims database of Taiwan, we were unable to investigate non-modifiable (Apo-E genotype) and modifiable risk factors (such as smoking or level of education). Age, prevalence of comorbidity and Charlson index were higher in the anaesthesia group than in the control group. Although we adjusted the Cox regression model for these 
factors, adjusting solely for these confounding factors might not have fully controlled for group differences. Additionally, it is possible that patients who underwent surgery were followed up more frequently than individuals in the control group, which could have allowed more opportunities for dementia diagnosis. The possible occurrence of post-operative cognitive decline after surgery could also lead to early awareness and initiation of a diagnostic evaluation for dementia. Moreover, we included patients who underwent anaesthesia for the first time since 1995, and the people in both anaesthesia and control groups might have been exposed to anaesthesia and surgery earlier in life. However, the variability in these exposures is unlikely to differ between these two groups.

\section{Future research}

In conclusion, this nationwide, population-based study showed a significant association between anaesthesia with surgery and dementia. Our findings support the view that patients who undergo anaesthesia and surgery may be at increased risk of dementia. Although anaesthesia and surgery have provided immeasurable health and social benefits, our observations highlight the need for further studies to understand the association and causality between anaesthesia with surgery and subsequent dementia.

Pin-Liang Chen, MS, Department of Computer Science and Information Engineering National Taiwan University, Taipei; Chih-Wen Yang, MD, Department of Neurology, Taipei Veterans General Hospital, Su-Ao and Yuanshan Branch, and National YangMing University School of Medicine, Taipei; Yi-Kuan Tseng, PhD, Graduate Institute of Statistics, National Central University, Jhongli; Wei-Zen Sun, MD, PhD, Department of Anaesthesiology, National Taiwan University Hospital, Taipei, Taiwan; Jane-Ling Wang, PhD, Department of Statistics, University of California, Davis, California, USA Shuu-Jiun Wang, MD, National Yang-Ming University School of Medicine, and Department of Neurology, Neurological Institute, Taipei Veterans General Hospital, Department of Neurology, Neurological Institute, Taipei Veterans General Hospita
Taipei; Yen-Jen Oyang, PhD, Department of Computer Science and Information Engineering, National Taiwan University, and Graduate Institutes of Biomedical Electronics and Bioinformatics, National Taiwan University, Taipei; Jong-Ling Fuh MD, Department of Neurology, Neurological Institute, Taipei Veterans General Hospital, Taipei, and National Yang-Ming University School of Medicine, Taipei, Taiwan

Correspondence: Jong-Ling Fuh, Neurological Institute, Taipei Veterans General Hospital, Taipei, Taiwan. Email: jlfuh@vghtpe.gov.tw

First received 16 Aug 2012, final revision 2 Apr 2013, accepted 15 Apr 2013

\section{Funding}

The study was supported by grants from Taipei Veterans General Hospital (V101C-105, VGHUST101-G7-1-2), National Science Council support for the Centre for Dynamical VGHUST101-G7-1-2), National Science Council support for the Centre for Dynamical
Biomarkers and Translational Medicine, National Central University, Taiwan (NSC 1002911-I-008-001), support from the Brain Research Centre at National Yang-Ming University and a grant from the Taiwanese Ministry of Education's Aim for the Top university plan. J.L.W.'s work was supported, in part, by a National Institutes of Health gran (R01AG025218-01).

\section{References}

1 Eckenhoff RG, Johansson JS, Wei H, Carnini A, Kang B, Wei W, et al. Inhaled anesthetic enhancement of amyloid-beta oligomerization and cytotoxicity. Anesthesiology 2004; 101: 703-9.

2 Mandal PK, Fodale V. Isoflurane and desflurane at clinically relevant concentrations induce amyloid betapeptide oligomerization: an NMR study. Biochem Biophys Res Commun 2009; 379: 716-20.

3 Culley DJ, Baxter MG, Crosby CA, Yukhananov R, Crosby G. Long-term impairment of acquisition of a spatial memory task following isofluranenitrous oxide anesthesia in rats. Anesthesiology 2004; 100: 309-14.

4 Planel E, Richter KEG, Nolan CE, Finley JE, Liu L, Wen Y, et al. Anesthesia leads to tau hyperphosphorylation through inhibition of phosphatase activity by hypothermia. J Neurosci 2007; 27: 3090-7.

5 Xie Z, Culley DJ, Dong Y, Zhang G, Zhang B, Moir RD, et al. The common inhalation anesthetic isoflurane induces caspase activation and increases amyloid beta-protein level in vivo. Ann Neurol 2008; 64: 618-27.

6 Wei $\mathrm{H}$, Xie Z. Anesthesia, calcium homeostasis and Alzheimer's disease. Curr Alzheimer Res 2009; 6: 30-5.
7 Vanderweydea T, Bednarb MM, Formanc SA, Wolozin B. latrogenic risk factors for Alzheimer's disease: surgery and anesthesia. J Alzheimers Dis 2010; 22 (suppl 3): s91-104.

8 Lee TA, Wolozin B, Weiss KB, Bednar MM. Assessment of the emergence of Alzheimer's disease following coronary artery bypass graft surgery or percutaneous transluminal coronary angioplasty. J Alzheimers Dis 2005; 7: 319-24.

9 Selnes OA, Royall RM, Grega MA, Borowicz LM, Quaskey S, McKhann GM. Cognitive changes 5 years after coronary artery bypass grafting. Arch Neurol 2001; 58: 598-604.

10 Knopman DS, Petersen RC, Cha RH, Edland SD, Rocca WA. Coronary artery bypass grafting is not a risk factor for dementia or Alzheimer disease. Neurology 2005; 65: 986-90.

11 Gasparini M, Vanacore N, Schiaffini C, Brusa L, Panella M, Talarico G, et al A case-control study on Alzheimer's disease and exposure to anesthesia. Neurol Sci 2002; 23: 11-4.

12 Baranov D, Bickler PE, Crosby GJ, Culley DJ, Eckenhoff MF, Eckenhoff RG, et al. Consensus statement: First International Workshop on Anesthetics and Alzheimer's disease. Anesth Analg 2009; 108: 1627-30.

13 World Health Organization. International Classification of Diseases, Ninth Revision, Clinical Modification (ICD-9-CM). WHO, 2009.

14 Charlson ME, Pompei P, Ales KL, Mackenzie CR. A new method of classifying prognostic comorbidity in longitudinal studies: development and validation. J Chron Dis 1987; 40: 373-83.

15 Futterer CD, Maurer MH, Schmitt A, Feldmann RE, Kuschinsky W, Waschke KF. Alterations in rat brain proteins after desflurane anesthesia. Anesthesiology 2004; 100: 302-8.

16 Duthie A, Chew D, Soiza RL. Non-psychiatric comorbidity associated with Alzheimer's disease. QJM 2011; 104: 913-20.

17 Driver JA, Beiser A, Au R, Kreger BE, Splansky GL, Kurth T, et al. Inverse association between cancer and Alzheimer's disease: results from the Framingham Heart Study. BMJ 2012; 344: e1442.

18 Zuo C, Zuo Z. Spine surgery under general anesthesia may not increase the risk of Alzheimer's disease. Dement Geriatr Cogn Disord 2010; 29: 233-9.

19 Culley DJ, Baxter M, Yukhananov R, Crosby G. The memory effects of general anesthesia persist for weeks in young and aged rats. Anesth Analg 2003; 96 : 1004-9.

20 Bohnen N, Warner MA, Kokmen E, Kurland LT. Early and midlife exposure to anesthesia and age of onset of Alzheimer's disease. Int J Neurosci 1994; 77 : $181-5$.

21 Lee IH, Culley DJ, Baxter MG, Xie Z, Tanzi RE, Crosby G. Spatial memory is intact in aged rats after propofol anesthesia. Anesth Analg 2008; 107: 1211-5.

22 Palotas A, Reis HJ, Bogats G, Babik B, Racsmany M, Engvau L, et al. Coronary artery bypass surgery provokes Alzheimer's disease-like changes in the cerebrospinal fluid. J Alzheimers Dis 2010; 21: 1153-64.

23 Williams-Russo P, Sharrock NE, Mattis S, Szatrowski TP, Charlson ME. Cognitive effects after epidural vs general anesthesia in older adults. A randomized trial. JAMA 1995; 274: 44-50.

24 Seitz DP, Shah PS, Herrmann N, Beyene J, Siddiqui N. Exposure to general anesthesia and risk of Alzheimer's disease: a systematic review and meta-analysis. BMC Geriatr 2011; 11: 83-90.

25 Farag E, Chelune GJ, Schubert A, Mascha EJ. Is depth of anesthesia, as assessed by the Bispectral Index, related to postoperative cognitive dysfunction and recovery? Anesth Analg 2006; 103: 633-40.

26 Gardner BK, O'Connor DW. A review of the cognitive effects of electroconvulsive therapy in older adults. J ECT 2008; 24: 68-80.

27 Bohnen NI, Warner MA, Kokmen E, Beard CM, Kurland LT. Alzheimer's disease and cumulative exposure to anesthesia: a case-control study. J Am Geriatr Soc 1994; 42: 198-201.

28 Abildstrom $\mathrm{H}$, Rasmussen LS, Rentowl $\mathrm{P}$, Hanning $\mathrm{CD}$, Rasmussen $\mathrm{H}_{\text {, }}$ Kristensen PA, et al. Cognitive dysfunction 1-2 years after non-cardiac surgery in the elderly. Acta Anaesthesiol Scand 2000; 44: 1246-51.

29 Avidan MS, Searleman AC, Storandt M, Barnett K, Vannucci A, Saager L, et al. Long-term cognitive decline in older subjects was not attributable to noncardiac surgery or major illness. Anesthesiology 2009; 111: 964-70.

30 Wan $Y, X u$ J, Ma D, Zeng Y, Cibelli M, Maze M. Postoperative impairment of cognitive function in rats: a possible role for cytokine-mediated inflammation in the hippocampus. Anesthesiology 2007; 106: 436-43.

31 Tang JX, Baranov D, Hammond M, Shaw LM, Eckenhoff MF, Eckenhoff RG. Human Alzheimer and inflammation biomarkers after anesthesia and surgery. Anesthesiology 2011; 115: 727-32.

32 Cibelli M, Fidalgo AR, Terrando N, Ma D, Monaco C, Feldmann M, et al. Role of interleukin-1beta in postoperative cognitive dysfunction. Ann Neurol 2010; 68: $360-8$. 